\title{
Effect of combined temperature-drought stresses on antioxidant activity of plants
}

Kurmanbayeva A.B.*, Yermukhambetova R.Zh., Bekturova A.Zh., Amanbayeva U.I., Gadilgereyeva B.Zh., Omarov R.T., Masalimov Zh.K.

L.N.Gumilyov Eurasian National University, Nur-Sultan, Kazakhstan

*e-mail: asylai-88@mail.ru

Altering climatic conditions and drought stress drastically affects the crop yield in agriculture. Plants respond and adapt to these abiotic stresses invariably by complex mechanisms inducing various morphological, biochemical, physiological, and molecular aspects. Additionally, it is known, that abiotic stresses results in the excessive production of Reactive Oxygen Species (ROS), leading to oxidative stress. Plants have evolved a wide range of enzymatic and non-enzymatic mechanisms to scavenge ROS and protect their cells against oxygen toxicity. However, the role of the antioxidant defense involving ROS-scavenging enzymes in the tolerance of barley plants to combined drought and temperature stresses is currently unknown.

The aim of the present work was to determine the importance of the modulation of the antioxidant system in barley to the combination of drought with high $\left(+40{ }^{\circ} \mathrm{C}\right)$ and low $\left(+10{ }^{\circ} \mathrm{C}\right)$ temperatures. To achieve this, plants were exposed to combined stresses for 5 days. Indeed, morphological parameters of the barley plants showed less root and shoot biomass accumulation and higher chlorophyll degradation compare to their controls. Further we checked the activity of the CAT and aldehyde oxidases (AOs) activities, where catalase (CAT) directly converts $\mathrm{H}_{2} \mathrm{O}_{2}$ into $\mathrm{H}_{2} \mathrm{O}$ and $\mathrm{AO}$ may participate in stress responses, because it catalyzes the oxidation of abscisic aldehyde to ABA, in the last step of ABA synthesis. 